\title{
Interview mit Prof. Jin Zhao金钊, TCM-Universität Chengdu - zur Stellung der klassischen Chinesischen Medizin und zu ihrer heutigen Anwendung einschließlich der Behandlung von Covid-19
}

\author{
Jin Zhao 金钊1 \\ Online publiziert: 23. Februar 2022 \\ (c) The Author(s) under exclusive licence to Springer Medizin Verlag GmbH, ein Teil von Springer Nature 2022
}

\section{Zusammenfassung}

Das Interview mit Prof. Jin Zhao 金钊 wurde im November 2021 von Andrea Crämer und Sebastian Oppitz geführt. Darin erläutert Prof. Jin sein besonderes Interesse an den verschiedenen Denkschulen der TCM und geht auf deren Anwendung in der klinischen Praxis ein. Einen Schwerpunkt des Interviews stellen die Erfahrungen von Prof. Jin mit der Behandlung von Covid-19 dar.

Schlüsselwörter Professor Jin Zhao · Klassische Chinesische Medizin · Denkschulen der Chinesischen Medizin . „Mitten-Denkschule“ · Differenzierung der Symptomkonfigurationen · Covid-19

\section{Interview with Prof. Jin Zhao金钊一the position of Classical Chinese Medicine and its modern applications including the treatment of COVID-19}

\begin{abstract}
In this interview, conducted by Andrea Crämer and Sebastian Oppitz in November 2021, Prof. Jin Zhao 金钊 talks about his medical background and his particular relationship to the different schools of thought in Chinese Medicine (gejia хиеshиo 各家学说) and how he puts this knowledge into clinical practice. An important part of this interview are Prof. Jin's experiences in treating COVID-19 diseases.
\end{abstract}

Keywords Professor Jin Zhao · Classical Chinese Medicine · Different schools of thought · "School of Spleen and Stomach Systems" · Pattern identification (bianzheng 辨证) · COVID-19

Andrea Crämer (AC): Professor Jin Zhao, Sie haben Ihren Ph.D. in Geschichte der TCM und klassische Schriften gemacht und unterrichten den Kurs zum Thema „Verschiedene Denkschulen der TCM“ (gejia xиeshuo 各家学说) auch an der Chengdu TCM-Universität. Gleichzeitig sind Sie ein bekannter Kliniker der jüngeren Generation und behandeln

Geführt von Andrea Crämer, Nürnberg, und Sebastian Oppitz, München

Aus dem Chinesischen übersetzt von Andrea Crämer, Nürnberg

Jin Zhao 金钊

3500008@qq.com

1 Chengdu TCM-Universität, Liutai dadao

Nr. 1166, 610075 Chengdu, Sichuan, Volksrepublik China bis zu 100 Patienten am Tag, wie ich das in der Ambulanz einer berühmten Apotheke in Chengdu selbst miterleben durfte. Wie sind Sie zur TCM und insbesondere zur Geschichte der TCM gekommen?

Prof. Jin Zhao 金钏 (JZ): Mein Weg zur TCM wurde sehr stark durch meine Familie beeinflusst und der Tatsache, dass mein Vater, Jin Zhilin 金治林, ebenfalls TCM-Arzt für Kardiologie, Diabetologie und Infertilität ist. Mich beeindruckte die tiefe Dankbarkeit, die die Patienten meinem Vater entgegenbrachten, so sehr, dass ich mich in der Oberstufe entschloss, TCM zu studieren, obwohl ich zunächst lieber Umweltschutz oder Journalismus studiert hätte. Der Lehrer meines Vaters empfahl mir, meinen Masterabschluss bei Professor Song Xing 宋兴 im Fachgebiet „Geschich- 


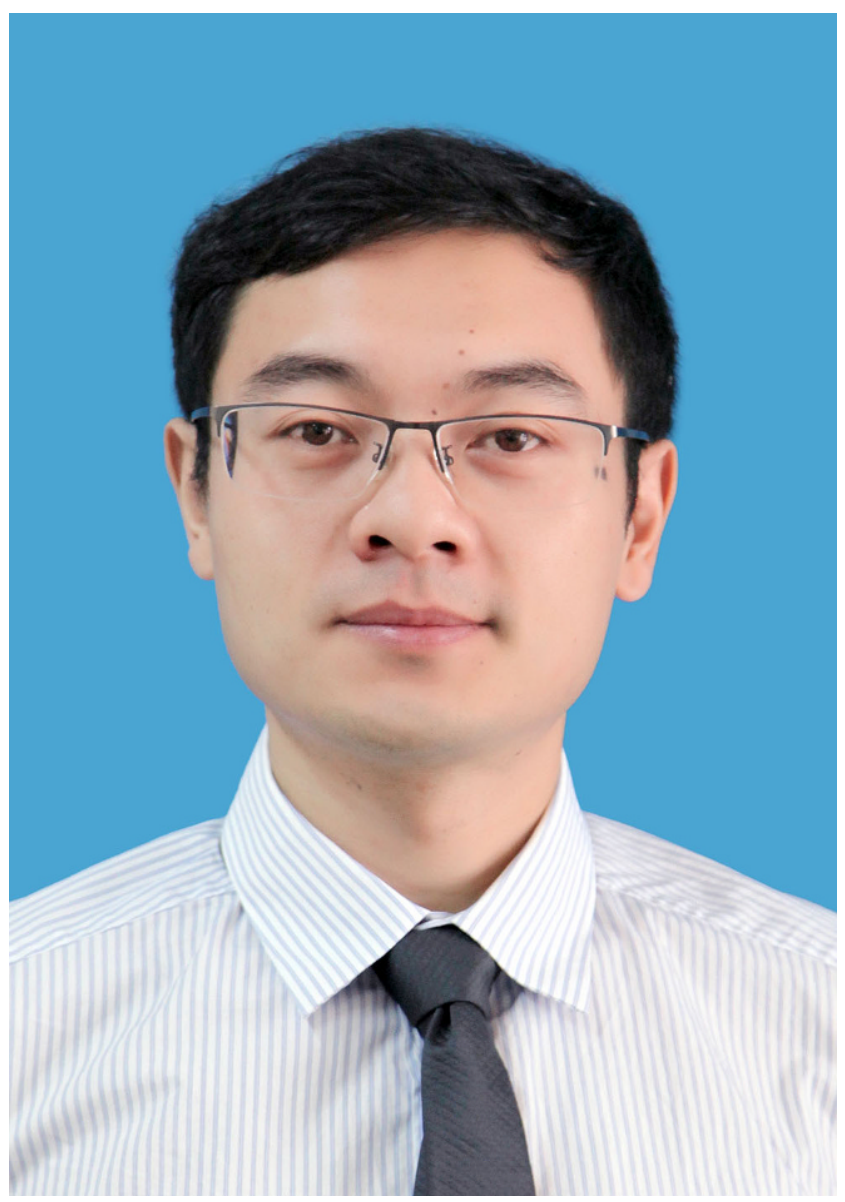

Abb. 1 Portraitaufnahme von Prof. Jin Zhao (Foto: Jin Zhao)

te der TCM und klassische Schriften" zu absolvieren, da er Studenten bevorzugte, die eine gute literarische Ausbildung vor allem in den klassischen Schriften hatten. Mein Vater legte großen Wert darauf, dass ich die Grundlagen der TCM sehr gut beherrschte und mit Fachliteratur früh in Verbindung kam, aber er ließ mich nicht häufig in seiner Praxis mitarbeiten, sondern erzählte mir immer von einigen spannenden Fällen, die ich dann aus bestimmten Gesichtspunkten bearbeiten sollte. Den Rest der Zeit verbrachte ich damit zu lernen. Außerdem erinnerte mich mein Vater stets daran, dass ich die Behandlung gemäß der Differenzierung der Symptomkonfigurationen unbedingt tiefgreifend beherrschen sollte und nicht ,beim bloßem Anblick von calor (,Hitze', re) gleich calor (,Hitze', re) kühle oder bei Anblick von Xue gleich Xue zurückhalte“.

Mein Betreuer Professor Song schätzte den folgenden Satz des Gelehrten Zhu Xi 朱喜 (1130-1200) sehr: „Der Weg des Lernens besteht darin, zuerst das (zugrundeliegende) Ordnungsprinzip ( $l i$ ) zu verstehen." Denn was seine klinischen Erkenntnisse und das, was er lehrte, anging, so waren Wissen und Praxis im Einklang; er sagte nie das eine, um dann das andere zu tun. Die Fülle seines Wissens und seiner Erfahrung, seine klare und eindeutige Beurtei- lung von Behandlung und Prognose von oft auch schweren Erkrankungen führten dazu, dass er unter seinen Kollegen sehr einflussreich wurde.

AC: Können Sie uns erklären, wie die verschiedenen Denkschulen im Laufe der Geschichte entstanden sein können? Ihre Rezepturen basieren häufig auf den Werken Li Dongyuans 李东垣 (oft auch Li Gao 李杲 genannt, 1180-1251), dem Begründer der „Mitten-Denkschule“.

JZ: Die unterschiedlichen Denkschulen resultierten nicht aus höherer Eingebung, sondern ihre Entstehung wurde durch den unermüdlichen Prozess des wiederholten Aufeinandertreffens von Theorie und Praxis ermöglicht.

Die verschiedenen Denkschulen haben einige Gemeinsamkeiten. Sie weisen jeweils ein theoretisches System, diagnostische Kriterien, eine Theorie über Arzneimittel und Rezepturen und deren Anwendung auf. Da sie aber auch unterschiedliche Ausgangspunkte, Standpunkte und Ergebnisse haben, sind diese Unterschiede auch faktische Realität.

Die Entstehung der Denkschulen kann man vor dem Hintergrund der verschiedenen geographischen, historischen, gesellschaftlichen Gegebenheiten sowie auch der aktuellen Erkrankungen sehen, aber man kann sie nicht ausschließlich auf diese beschränken.

Bei der TCM spielt die Differenzierung der Symptomkonfigurationen (bianzheng 辨证) die wichtigste Rolle, denn ihre Art bestimmt die Behandlungsmethode und damit die Rezepturwahl. Dabei handelt es sich um einen strengen Ablauf von logischer Analyse und Argumentation, der sich von Diagnose bis zur Behandlung durchzieht.

Sebastian Oppitz (SO): Können Sie für Therapeuten ohne spezifische Kenntnisse in wenigen Sätzen die Kernpunkte der „Mitten-Denkschule“ umreißen?

JZ: Li Dongyuans 李东垣 Theorie und seine wissenschaftlichen Erkenntnisse sind in der Tat sehr wertvoll. Er untersuchte in seiner Denkschule namens ,die Wandlungsphase Erde suppletierende Denkschule“ (butupai 补土派) das intrinsische System der oo. lienalis et stomachi (Fk „Milz“ und „Magen“, pi wei) und deren Verbindungen mit den anderen vier oo. horreales (Speicherfunktionskreise, zang). Dieses System hat den größten Einfluss auf unsere Physiologie und wird deshalb auch die „erworbene Konstitution“ genannt. Die Regeneration aller großen Funktionskreissysteme im Körper ist von der erworbenen Konstitution abhängig. Dies gilt auch bei Erkrankungen, die durch äußere Heteropathien (Schrägläufigkeiten, xie) verursacht werden. Hier wird einer Ausleitung der Heteropathie (Schrägläufigkeit, xie) eine Stärkung des qi orthopaticum (geradläufiges Qi, zhengqi) vorgezogen. 
Li Dongyuans zentraler Lehrsatz lautet: „Wenn die oo. lienalis et stomachi (Fk ,Milz' und ,Magen“, pi wei) im Inneren geschädigt werden, können daraus alle (einhundert) Erkrankungen hervorgehen." Dies bedeutet, dass die Entwicklung vieler Erkrankungen direkt oder indirekt mit dem Zustand der oo. lienalis et stomachi (Fk ,Milz“ und „Magen“, pi wei) zusammenhängen und auch von diesen direkt oder indirekt beeinflusst werden kann. Eine innere Schädigung der oo. lienalis et stomachi (Fk „Milz“ und „Magen“, pi wei) kann zu einer Vielzahl an Erkrankungen der Augen, Nieren, des Herz-Kreislauf-Systems, der Lunge sowie auch Neoplasmen führen.

Eine andere bemerkenswerte Entdeckung war, dass eine depletio qi (energetische Schwäche des Qi, qixu) großen calor („Hitze“, re) hervorrufen kann, was auch als Yin-ardor (Yin-,,Glut“, yinhuo) bekannt ist, Daraus resultierte das Therapiekonzept ,Warmes und Süßes können großen $c a$ lor (,Hitze‘, re) beseitigen“. (Anmerkung der Übersetzerin: Hier ist mit ,groß“ nicht unbedingt hohes Fieber gemeint. Es handelt sich vielmehr um ein starkes Hitzegefühl, das vom Patienten bei normaler Körpertemperatur bis hin zum hohem Fieber empfunden wird.) Dies führte zu einem verbesserten Verständnis und einer effektiveren Behandlung einiger morbi varii (verschiedene Krankheiten, zabing). Des Weiteren inspirierte Li Dongyuans Theorie die später aufkommende ,Denkschule des Wärmens und des Suppletierens“ (wenbu xuepai 温补学派) mit ihrem Fokus auf dem o. renalis (Fk „Niere“, shen).

Alles in allem hatte seine Theorie einen großen Einfluss auf nachfolgende Ärztegenerationen und konnte die Qualität der Behandlung in allen Disziplinen deutlich verbessern.

SO: Welche Beschwerdebilder lassen sich Ihrer Meinung nach besonders gut mit den Konzepten der ,Mitten-Denkschule" therapieren?

JZ: Alle Beschwerdebilder, deren Krankheitsmechanismus sich in dem vorliegenden Stadium mit dem einer inneren Schädigung der oo. lienalis et stomachi (Fk ,Milz“ und „Magen“, pi wei) deckt. Dies ist eine Verdeutlichung des Prinzips ,,verschiedene Erkrankungen mit der gleichen Therapie behandeln“.

Wir sollten uns aber nicht von vermeintlich eindeutigen Symptomen dazu verleiten lassen zu denken, dass sie immer zwangsläufig eine innere Schädigung der oo. lienalis et stomachi (Fk „Milz“ und „Magen“, pi wei) darstellen. Manchmal können auch solche Symptome zu ganz anderen Symptomkonfigurationen gehören und bedürfen einer anderen Behandlung.

SO: Wie sieht Ihr Alltag in der Praxis aus? Welche Rezepturen nutzen Sie besonders häufig? Werden in diesen Rezepturen besondere Arzneimittel, die sonst nicht häufig verwendet werden, verschrieben, oder werden besondere Paozhi-Verfahren genutzt?

JZ: In der Praxis behandle ich ein breites Spektrum an Erkrankungen wie z.B. Fieber und Allergien im Kindesalter, Gastritiden, Menstruationsstörungen, PCOS, Infertilität, Nephritiden, Krebsleiden und auch viele andere Erkrankungen, für die die westliche Medizin entweder keine Erklärung findet oder die von ihr nicht zufriedenstellend behandelt werden können. Welche Theorie und welche Rezepturen ich jeweils einsetze, ergibt sich aus der Zusammensetzung der verschiedenen Symptomkonfigurationen meiner Patienten und Patientinnen.

Da mein Spezialgebiet die verschiedenen Denkschulen der CM sind, kann ich aus dem Erfahrungsschatz zahlreicher früherer Ärzte schöpfen. Besonders häufig basieren meine Rezepturen auf der „Abhandlung über schädigende Kälte (algor laedens)“ (Shanghan lun, von Zhang Zhongjing, aus dem 2. Jh.), den Klassikern von Li Dongyuan, der Lehre von den morbi temperati (,Wärme“-Erkrankungen, wenbing), den Aufsätzen über calor-humidus-Erkrankungen (,Feuchtigkeit-Hitze“, shire) und auf Theorien anderer Ärzte. Ich bevorzuge einfache Arzneimittel, die häufig verwendet werden, damit meine Patienten ihre Arzneimittel überall einfach beschaffen können.

Außerdem lege ich großen Wert auf die Grundlagen der TCM bei der Auswahl von Arzneimitteln bezüglich des Temperaturverhaltens, der Geschmacksrichtung, der Wirkrichtung (Emporheben, Absenken, Aus- und Eintreten), des Leitbahn-/Funktionskreisbezugs usw. Diese Regeln erlauben eine einfache Auswahl und das einfache Austauschen von Arzneimitteln; daher nutze ich nur sehr wenige sehr spezielle Arzneimittel, die in Europa sowieso häufig verboten sind.

AC: Wie ich in Ihrer Praxis sehen konnte, verschreiben Sie jedoch nicht nur ausschließlich auf Grundlage der ,MittenDenkschule“. Welchen Stellenwert nehmen Ihrer Meinung nach hier die Symptomkonfigurationen und eine differenzierte Therapie ein? Hätten Sie ein kurzes Fallbeispiel als Demonstration?

JZ: Welche Theorien meine klinische Behandlung leiten und ob ich diese anwende oder nicht (z.B. die ,die Wandlungsphase Erde suppletierende Denkschule“), hängt nicht von meiner persönlichen Präferenz ab, sondern von der $\mathrm{Na}$ tur der Erkrankung zum Behandlungszeitpunkt. Im philosophischen Sinne gesagt: Die Entwicklung objektiver Dinge folgt nicht dem Willen des Einzelnen. Wir müssen den Gesetzen der Krankheitsentwicklung folgen, und entsprechend dem gegenwärtigen Zustand werden die Behandlungsmethode und die Rezeptur gewählt. 
Abb. 2 Prof. Jin Zhao bei der Pulstastung einer Patientin (Foto: Andrea Crämer)

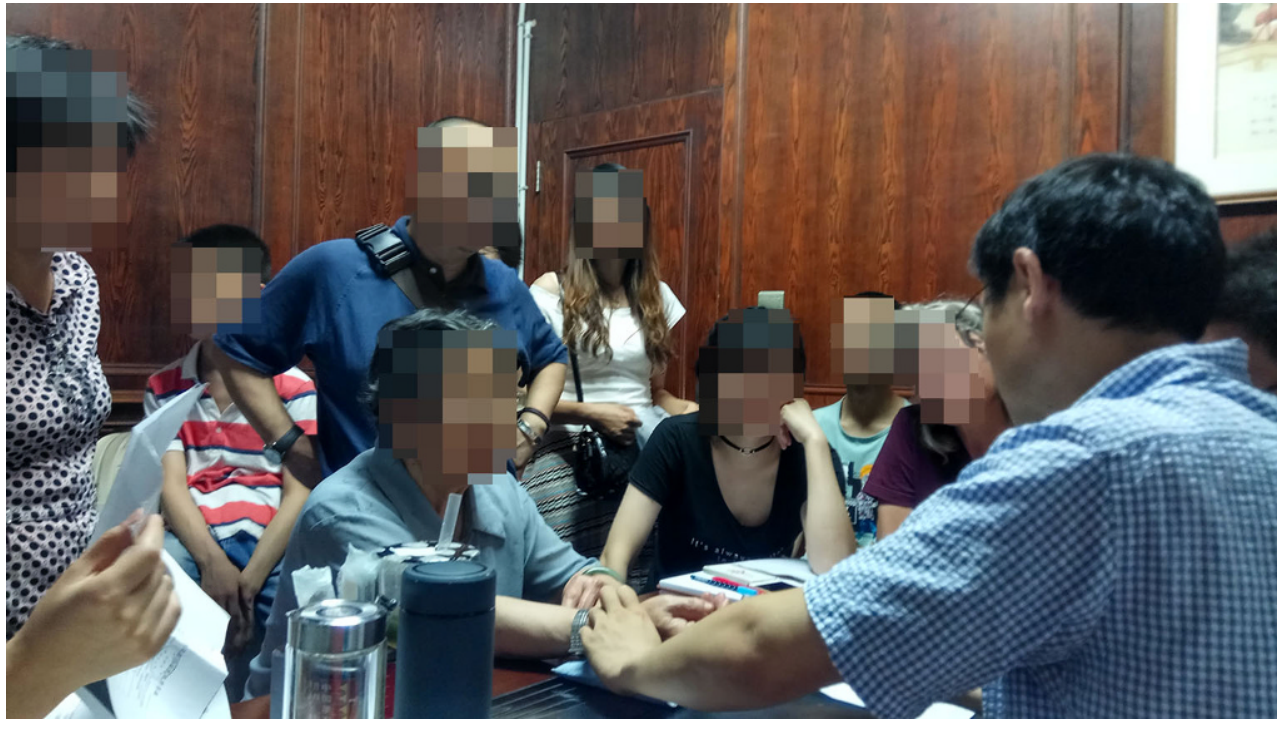

Unser persönlicher Wille kann nicht der Natur der Erkrankung aufoktroyiert werden, denn so würden wir das Prinzip der Differenzierung der Symptomkonfigurationen hintergehen. Die CM ist eine objektive, rationale und logische Disziplin. Dieses Leitprinzip hat es der CM ermöglicht, sich von rein empirischen Zusammenfassungen und von mechanistischen Vergleichen vorangegangener Erfahrungen zu lösen und zu einer wissenschaftstheoretischen Medizin zu werden.

Erst ein grundlegendes Verständnis der Differenzierung der Symptomkonfigurationen ermöglicht es uns, dem anderen Grundcharakteristikum der TCM, nämlich der Ganzheitlichkeit (zhengti 整体), gerecht zu werden. Nur wenn beide Kriterien beachtet werden, kann die TCM eine überlegene Position in der Behandlung einnehmen, wenn die westliche Medizin sie nicht beanspruchen kann.

So wird zum Beispiel bei atrophischen Krankheiten und occlusio-Erkrankungen (Bi-Syndrome, bizheng) oft nur eine depletio qi et xue (energetische Schwäche von Qi und Xue, qi xиe $x u$ ) oder eine Blockade durch humor algidus („Kälte-Feuchtigkeit“, hanshi) in Erwägung gezogen, wobei es durchaus mehr Symptomkonfigurationen gibt, die diese Erkrankungen hervorrufen können, wie uns das folgende Beispiel zeigt.

Eine Patientin, ca. 60 Jahre, kam in meine Praxis, da sie seit drei Monaten ihre Beine nicht mehr bewegen konnte und eine schmerzhafte Steifigkeit in den Hüft- und Kniegelenken aufwies. Bei ihrem ersten Besuch musste sie sogar stehen, da sie sich nicht hinsetzen konnte. Bei der Diagnose fiel eine stark belegte Zunge auf. Während andere Kollegen sie zuvor mit Rezepturen aus der „Abhandlung über schädigende Kälte (algor laedens)“ (Shanghan lun), der „wärmenden und suppletierenden Denkschule“ und der „,die Wandlungsphase Erde suppletierenden Denkschule" oder mit Rezepturen, die die oo. hepaticus et renalis (Fk „Leber“ und „Niere“, gan shen) suppletieren, humor algidus („Kälte-Feuchtigkeit", hanshi) zerstreuen, das Xue dynamisieren und die reticulares („Netzleitbahnen“, luo) durchgängig machen usw. behandelten, wurde ihr Zustand zunehmend schlechter, bis ihre Beine vollkommen bewegungsunfähig wurden. Bei genauerer Analyse der Symptomkonfigurationen konnten wir hier einen calor humidus („FeuchtigkeitHitze“, shire) feststellen, der durch die vorangegangenen Behandlungen nicht nur nicht gelindert, sondern auch noch verschlimmert wurde.

Entsprechend des Krankheitsmechanismus - die reticulares („Netzleitbahnen“, lиo) blockierender calor humidus („Feuchtigkeit-Hitze“, shire) - haben wir aromatische humor („Feuchtigkeit“", shi) umwandelnde, ventus (,Wind“, feng) herauslösende, zerstreuende und durchlässig machende, humor („Feuchtigkeit“", shi) ausleitende, die reticulares („Netzleitbahnen“, luo) durchgängig machende Arzneimittel genutzt. Das waren hier das „Dekokt der drei Samen“ (Sanren tang) und eine Modifikation des „Dekoktes mit Agastache, Magnolia, Pinellia und Poria“ (Huopo xialing tang), und nach dreiwöchiger Therapie verschwanden die Symptome in ihren Beinen, und sie konnte sich wie gewohnt bewegen. Dies zeigt die wahre Stärke der Differenzierung der Symptomkonfigurationen.

Ein anderes Beispiel zeigt die mechanistische Wahl von lediglich zwei Rezepturen bei allergischer Purpura auf. Hier werden das „Pulver mit Lonicera und Forsythia“ (Yinqiao san) und das „Dekokt mit Bubali cornu und Rehmannia“ (Xijiao dihuang tang) im Grunde genommen nur aufgrund des Krankheitsnamens und der Krankheitsdifferenzierung aus Sicht der westlichen Medizin verschrieben. Bei Differenzierung der Symptomkonfigurationen stellen wir aber oft fest, dass häufig eine Behandlung mit humor („,Feuchtigkeit", shi) umwandelnden und die extima (Oberfläche, biao) lösenden oder mit scharfen, wärmenden, die extima (Ober- 
fläche, biao) lösenden oder sogar die Mitte erwärmenden und humor (,Feuchtigkeit“", shi) umwandelnden Rezepturen nötig ist. Dieser Therapieansatz unterscheidet sich grundlegend in der Symptomkonfiguration und der Zuordnung nach den Acht Leitkriterien der beiden zuvor genannten Rezepturen.

Diese beiden Beispiele sollten uns Anlass geben, tiefgründiger über unsere Therapien nachzudenken, da sonst eine weitere Entwicklung der TCM gefährdet ist. Die Differenzierung der Symptomkonfigurationen ist nicht nur die Grundlage, die zu Beginn des TCM-Studiums vermittelt werden muss, sondern auch das höchste Niveau, welches wir in unserer praktischen Tätigkeit erreichen müssen. Sie ist die Seele der klinischen TCM!

AC: Als ich miterleben durfte, wie Sie Ihre Patienten behandelten, gaben Sie ihnen öfters kurze diätetische Ratschläge. Welchen Stellenwert geben Sie der Diätetik in Ihrer Therapie?

JZ: Die Ernährung ist bei unserer Behandlung sehr wichtig, da sie zuerst in den Magen gelangt und sich damit direkt auf die Funktion der oo. lienalis et stomachi (Fk „Milz“ und „Magen“, pi wei) auswirkt.

Außerdem haben auch alle Lebensmittel unterschiedliche Temperaturverhalten, Geschmacksrichtungen und Wirkrichtungen, auch wenn sie im Vergleich zu den Arzneimitteln aus unseren Rezepturen viel weniger stark ausgeprägt sind. Wenn der Mensch erkrankt, nehmen alle Funktionen des Körpers ab, und die Stoffwechselkapazität ist reduziert. Dann sind die verschiedenen Eigenschaften der Nahrungsmittel relativ stärker ausgeprägt, und wenn diese unserer Behandlungsrichtung widersprechen, so können sie sich nicht nur auf die Wirksamkeit der Behandlung auswirken, sondern sogar zu einer Verschlechterung der Erkrankung führen. Daher sind diätetische Gebote und Verbote ein wichtiger Teil unserer Behandlung.

Wenn zum Beispiel Husten durch eine Erkältung verursacht wird, dann sollte die Behandlung darin bestehen, eine antitussive Wirkung durch Zerstreuen von algor („Kälte“, han) zu erzielen. Viele Patienten (in China) greifen jedoch zu Birnen, Weißmorcheln (Yin'er) und Fritillaria (Beimu), was letztendlich das Gegenteil unserer Behandlungsrichtung darstellt und die Wirkung nicht nur verringert, sondern sie behindert.

Oft wird Tumorpatienten geraten, Eiweißpräparate, suppletierende Arzneimittel und viel Fleisch zu sich zu nehmen, um ihr Immunsystem zu stärken. Doch leider leiden diese Patienten schon oft von vornherein an einer depletio (energetische Schwäche, $x u$ ) des o. lienalis (Fk „Milz“, pi) mit humor algidus (,Kälte-Feuchtigkeit", hanshi), welcher die Mitte blockiert. So reduziert sich deren Verdauungsfunktion weiter, der trübe humor („Feuchtigkeit“, shi) staut sich ein und stagniert, was zur Einstauung und zur Blockade des Qi-Mechanismus führt, wobei die oo. lienalis et stomachi (Fk „Milz“ und „Magen“, pi wei) in ihrer Funktion nur weiter eingeschränkt werden, was sogar bis zum Tod führen kann.

AC: In Sichuan dominiert ein besonders feuchtes Klima alle Jahreszeiten, was Ihre Therapie maßgeblich beeinflusst. Sie haben bereits auch in Amerika für eine längere Zeit praktiziert und konnten dort die Ansätze der ,Mitten-Denkschule“ für Ihre Behandlung erfolgreich nutzen. Was können wir von Ihrem Ansatz im Westen und speziell in Deutschland lernen?

JZ: Das Klima in Sichuan ist relativ feucht, die Ernährungsgewohnheiten unterscheiden sich signifikant vom Rest Chinas, des Weiteren ist die Konstitution der Einwohner Sichuans auch eine andere. Somit sehe ich in meiner Praxis oft, dass in Kombination mit der eigentlichen Erkrankung mit humor (,Feuchtigkeit“", shi) auch begleitend algor (,Kälte“, han) und wässrige pituita (wässriger ,Schleim“, yin) auftreten. $\mathrm{Zu}$ den häufig behandelten Symptomkonfigurationen gehören humor algidus („Kälte-Feuchtigkeit“, hanshi), der das mittlere Calorium (mittlerer Wärmebereich, zhongjiao) blockiert, calor humidus (,FeuchtigkeitHitze“, shire), der den o. tricalorii (Fk „Drei Wärmebereiche“, sanjiao) angreift, Defizienz des qi lienale (Qi des Fk „Milz“, piqi) oder depletive Schädigung des yang lienale (Yang des Fk „Milz“, piyang). Daraus ergibt sich meine Auswahl an Arzneimitteln, und ich habe damit in der Regel guten Erfolg.

Als ich in den Vereinigten Staaten praktizierte, hielt ich mich an die gleichen Prinzipien. Wie ich bereits erwähnte, nutze ich die Behandlungsmethoden Li Dongyuans, sobald es sich mit einer inneren Schädigung der oo. lienalis et stomachi (Fk „Milz“ und „Magen“, pi wei) in Einklang bringen lässt. Seine Rezepturen sind vielfältig einsetzbar.

Ich denke, dass die Differenzierung der Symptomkonfigurationen ein universeller Ansatz der TCM ist, der gilt, egal, ob man sich in Deutschland, Amerika, China, Afrika, Australien oder sogar der Antarktis befindet.

Ich habe gehört, dass erstens das Klima in Deutschland relativ kalt ist und zweitens die Ernährung auch eher kalt, süß und fettig ist. Deswegen gehe ich davon aus, dass innere Schädigungen der oo. lienalis et stomachi (Fk „Milz“ und „Magen“, pi wei) sehr wahrscheinlich sind und damit eine breite Anwendung sowohl der „Denkschule des algor laedens (schädigende ,Kälte‘, shanghan)“ als auch der Li Dongyuans möglich ist.

SO: Die letzten beiden Jahre sind v. a. hier im Westen von der Corona-Pandemie gezeichnet gewesen. Haben Sie während der Pandemie akute Corona-Infektionen und Long- 
Covid-Fälle behandelt? Wenn ja, wo sehen Sie die hauptsächlichen Pathomechanismen, und haben Sie Tipps für die Behandlung über die offiziellen chinesischen Leitlinien hinaus?

JZ: Seit Beginn der Corona-Pandemie habe ich selbst ca. 100 und unsere kleine Gruppe hat ca. 200 Patienten behandelt.

Es gibt hauptsächlich zwei Behandlungsphasen, nämlich die Akut- und Rekonvaleszenzphase. Bei unseren Fällen konnten wir als Gemeinsamkeit Epidemisch-Toxisches (yi$d u$ ) und trüben humor (,Feuchtigkeit“, shi) feststellen.

Die Ursache der Erkrankung ist Epidemisch-Toxisches (yidu), dessen Kernelement humor-Toxisches (,Feuchtigkeit"-Toxisches, shidu) ist, wobei eine Dysfunktion der Funktion der oo. lienalis et stomachi (Fk „Milz“ und „Magen“, pi wei) die Hauptursache der Entstehung der humorHeteropathie („Feuchtigkeit"-Schrägläufigkeit, shixie) darstellt.

Die inneren Dysfunktionen der Funktionskreise in Kombination mit den äußeren ungünstigen klimatischen Gegebenheiten bieten eine gute innere und äußere Grundlage für das Eintreten von Epidemisch-Toxischem (yidu). Sobald das Epidemisch-Toxische die Funktionskreise affiziert hat und sich mit dem humor-Toxischen (,Feuchtigkeit“-Toxisches, shidu) verbindet, kann die Heteropathie (Schrägläufigkeit, xie) in den „Ursprung der Membran“ (moyuan 膜原 $)^{1}$ eintreten, sich im Körper verbreiten, in den $o$. tricalorii (Fk „Drei Wärmebereiche“, sanjiao) eintreten, sich in ariditas (,Trockenheit“, zao) umwandeln, die im oberen Calorium (oberer Wärmebereich, shangjiao) die reticulares („Netzleitbahnen“, luo) blockiert, das mittlere Calorium (mittlerer Wärmebereich, zhongjiao) wird durch trüben $h u$ mor („Feuchtigkeit“, shi) behindert, und der Qi-Mechanismus des o. tricalorii (Fk „Drei Wärmebereiche“, sanjiao) wird blockiert. Zu guter Letzt tritt die Heteropathie (Schrägläufigkeit, xie) in die reticulares (,Netzleitbahnen“, luo) ein, der gesamte Qi-Mechanismus kommt zum Erliegen, und der Patient stirbt.

Die Behandlung basiert auf der Entfaltung des o. pulmonalis (Fk „Lunge“, fei) sowie darauf, Toxisches herauszulösen, die reticulares (,Netzleitbahnen“, luo) durchgängig machen und pituita (,Schleim“, tan) umwandeln und die Heteropathien (Schrägläufigkeiten, xie) aus dem „Ursprung

\footnotetext{
${ }^{1}$ Der Begriff „Membran-Ursprung“ (moyuan 膜原) kann zwei Bedeutungen haben: Nach dem Kap. 39 der „Unbefangenen Fragen“ im „Inneren Klassiker des Gelben Fürsten“ (Huangdi Neijing Suwen, etwa 1. Jh. v.u.Z., im 8. und 11. Jh. überarbeitet) bezeichnet er den Bereich zwischen Pleura und Diaphragma. In der Schule der „Wärme"-Erkrankungen wird er als Bereich zwischen extima (Oberfläche, biao) und intima (Inneres, li) gesehen und gilt auch als „Pforte zum o. tricalorii (Drei Wärmebereiche, sanjiao)“, Anm. d. Übers., s. Liu Guohui. Warm Diseases. A Clinical Guide: Eastland Press, 2001:186.
}

der Membranen“ (s. oben) herauszulösen. Dabei werden Rezepturen wie der „Trank, der an die Quelle vordringen lässt“ (Dayuan yin), „Dreifach lösender Trank“ (Sanxiao yin $)^{2}$, das „Dekokt mit Agastache, Magnolia, Pinellia und Poria“ (Huopo xialing tang), „Dekokt der drei Körner mit Bupleurum“ (Chaihu sanren tang), das „Notopterygium-Dekokt, das Feuchtigkeit (humor) bezwingt" (Qianghuo shengshi tang), das „Pulver der fünf Ling-Bestandteile“ (Wuling san), das „Dekokt mit Ephedra, Prunus armeniaca, Gypsum und Glycyrrhiza“ (Maxing shigan tang), das „Die Mitte regulierende Dekokt" (Lizhong tang) usw. und deren Modifikationen verwendet.

Bei der Behandlung halten wir uns an das Prinzip der Differenzierung der Symptomkonfigurationen, achten auf die körperliche Verfassung des Patienten, seine Vitalität, ermitteln die Hauptursache des humor-Toxischen (,Feuchtigkeit"-Toxisches, shidu) und passen die Rezepturen flexibel an die Erkrankungsphase und den Patienten an.

Ich erinnere mich noch sehr gut daran, wie ein guter Freund von mir in Wuhan und seine Familie alle gleichzeitig mit COVID-19 infiziert waren: Seine Eltern waren schwer, er und seine im 6. Monat schwangere Frau leicht erkrankt. Wir haben alle vier mit vollkommen verschiedenen Behandlungsmethoden behandelt. Bei allen erfolgte eine folgenlose Ausheilung, und seine Ehefrau brachte eine gesunde Tochter zur Welt.

Ich denke, dass wir aufgrund der strikten Befolgung der Differenzierung der Symptomkonfigurationen sehr zufriedenstellende Ergebnisse erzielen konnten und lediglich einen Todesfall unter den Fällen, die wir insgesamt behandelten, zu beklagen hatten.

Bei der Behandlung dieser Erkrankung gibt es einige Punkte zu beachten:

1. Oft wird sie fälschlicherweise als algor laedens (schädigende „Kälte“, shanghan) diagnostiziert. Die Wahl der Arzneimittel sollte sich nicht auf hochdosierte algor („Kälte“, han) zerstreuende und die extima (Oberfläche, biao) lösende Arzneimittel stützen, sondern auf die aromatische Umwandlung des trüben humors (,Feuchtigkeit“, shi) sowie auf das Lösen und Durchgängigmachen des Qi-Mechanismus.

2. Bei wiederkehrendem oder sogar hohem Fieber, welches nicht abklingt, wird oft fälschlicherweise calorToxisches („Hitze“-Toxisches, redu) im splendor yang (,Überstrahlung des Yang“, yangming) diagnostiziert,

\footnotetext{
${ }^{2}$ Ingredienzen des „Dreifach lösenden Trankes“ (Sanxiao yin): Arecae semen (Binglang), Tsaoko fructus (Caoguo), Magnoliae cortex (Houpo), Paeoniae radix lactiflora (Baishao), Glycyrrhizae radix (Gancao), Anemarrhenae rhizoma (Zhimu), Scutellariae radix (Huangqin), Rhei rhizoma (Dahuang), Puerariae radix (Gegen), Notopterygii rhizoma (Qianghuo), Bupleuri radix (Chaihu), s. https://baike.baidu.com/item/ \%Е4\%B8\%89\%E6\%B6\%88\%E9\%A5\%AE/9761971.
} 
Abb. 3 Prof. Jin Zhao bei der Zungendiagnose einer Patientin (Foto: Andrea Crämer)

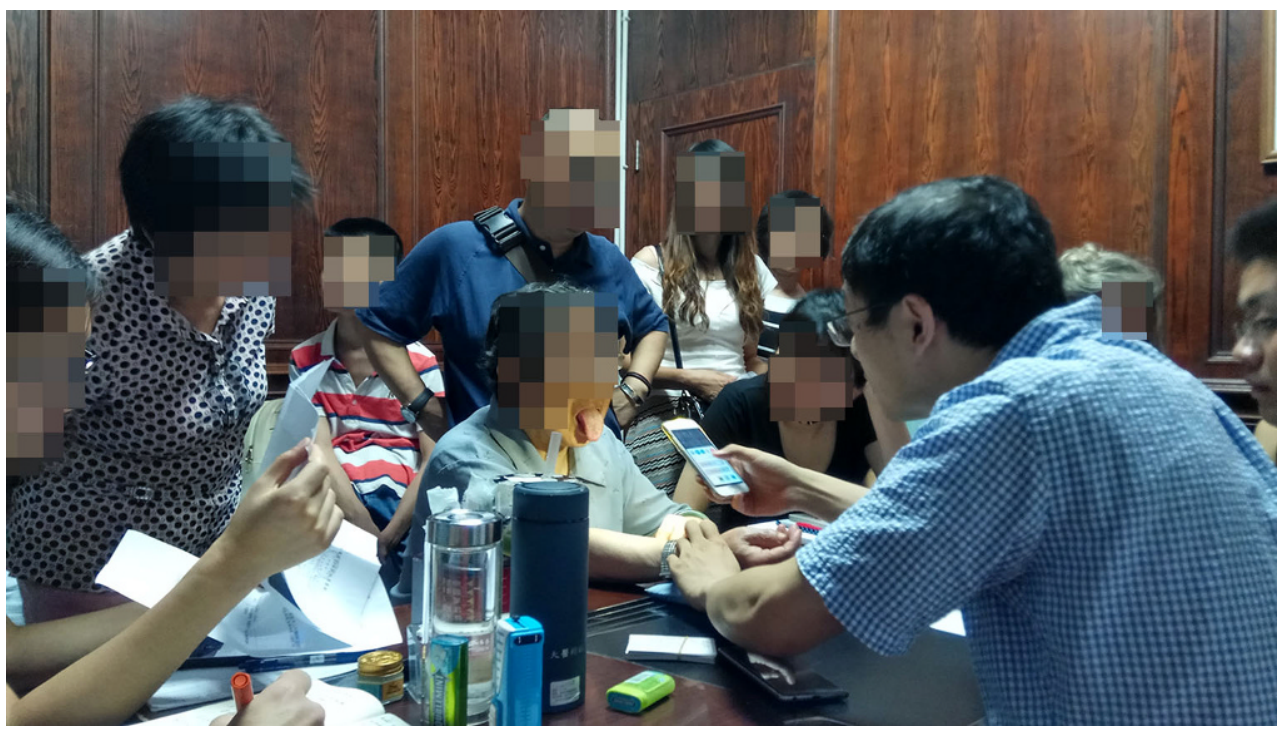

und kühle und kalte Arzneimittel werden verfrüht und in hoher Dosierung angewandt.

3. Bei Husten mit geringer Menge an Auswurf wird oft fälschlicherweise ariditas (,Trockenheit“, zao), die den o. pulmonalis (Fk „Lunge“, fei) schädigt, oder überhandnehmender calor („Hitze“, re), der die aktiven Säfte schädigt, diagnostiziert. Bei der Wahl der Arzneimittel sollte der Schwerpunkt nicht auf dem Klären des o. pulmonalis (Fk „Lunge“, fei) und dem Befeuchten der ariditas (,Trockenheit“, zao) oder dem Nähren von Yin und Klären von calor („Hitze“, re) liegen, sondern darauf, den humor („Feuchtigkeit“, shi) umzuwandeln und das Toxische herauszulösen, den $o$. pulmonalis (Fk „Lunge“, fei) zu entfalten und die reticulares („Netzleitbahnen“, lиo) durchgängig zu machen. Sobald der humor („,Feuchtigkeit“, shi) beseitigt und die reticulares („Netzleitbahnen“, luo) durchgängig sind, werden sich die aktiven und struktiven Säfte selbst wieder verteilen können, die ariditas („Trockenheit“, zao) wird gelöst, die aktiven Säfte werden zurückgeführt, und der calor („Hitze“, re) wird zurückgedrängt.

4. Bei Müdigkeit wird oft fälschlicherweise eine depletio $q i$ (energetische Schwäche des Qi, qixu) der oo. lienalis et pulmonalis (Fk „Milz“ und „Lunge“, pi fei) diagnostiziert, was zur blinden Anwendung von suppletierenden Arzneimitteln führt. Jedoch sollte hier die Behandlung nicht auf dem Suppletieren der oo. lienalis et pulmonalis (Fk „Milz“ und „Lunge“, pi fei) liegen, sondern darin, den humor („Feuchtigkeit“, shi) umzuwandeln und den Qi-Mechanismus zu entfalten und durchgängig zu machen. Sobald der humor („Feuchtigkeit“, shi) umgewandelt ist, kann sich auch das Qi umwandeln, und das Auf- und Absteigen des Qi-Mechanismus wird wiederhergestellt. Die Arzneimittel sollten nicht calor („Hitze“, re) kühlen und Toxisches herauslösen, sondern humor
(,Feuchtigkeit“, shi) umwandeln und die Heteropathie (Schrägläufigkeit, xie) herauslösen. Sobald der humor (,Feuchtigkeit“, shi) beseitigt ist, hat der calor (,Hitze“, $r e)$ keinen Raum mehr, sich zu entwickeln.

5. Bei Atemnot werden oftmals verfrüht und in zu hoher Dosierung Ephedrae herba (Mahuang), Gypsum fibrosum (Shigao), Lepidii/Descurainiae semen (Tinglizi) und andere den o. pulmonalis (Fk „Lunge“, fei) entfaltende, calor (,Hitze“, re) ausleitende, Keuchen stabilisierende Arzneimittel verwendet. Dabei sollte die Therapie hauptsächlich auf der Umwandlung von humor (,Feuchtigkeit“", shi), dem Entfalten des o. pulmonalis (Fk „Lunge“, fei), dem Durchgängigmachen der reticulares („Netzleitbahnen“, lио) und dem Herauslösen von Toxischem basieren.

6. Bei Sektionen fallen bei Verstorbenen vielfach Embolien auf. Aus TCM-Sicht handelt es sich um eingestautes Xue, welches die reticulares („Netzleitbahnen“, luo) blockiert. Hier sollte man nicht blind stark Xue dynamisierende und die reticulares („Netzleitbahnen“, lиo) durchgängig machende Arzneimittel verschreiben. Rezepturen wie „Phragmites-Dekokt“ (Weijing tang) oder Arzneimittel wie Chuanxiong rhizoma (Chuanxiong), Allii macrostemonis bulbus (Xiebai) und Curcumae longae tuber (Yujin) sollten nach gründlicher Abwägung der Versorgung des Körpers mit Qi und aktiven Säften verschrieben werden. Der Einsatz von niedrig dosierten das Xue dynamisierenden Arzneimitteln ist durchaus angezeigt, um die Durchblutung zu fördern, ohne dabei das $q i$ orthopaticum (geradläufiges Qi, zhengqi) zu zerstreuen.

Zusammenfassend lässt sich sagen, dass wir die Heteropathie (Schrägläufigkeit, xie) der Erkrankung, den toxischen humor („Feuchtigkeit“", shi), die Konstitution des Patienten 
und die humor-Heteropathie (,Feuchtigkeit“-Schrägläufigkeit, shixie) genau im Auge behalten müssen.

Die Grundsätze der Behandlung während der Rekonvaleszenzphase sind wie folgt:

1. Zur Umwandlung des humors (,Feuchtigkeit“", shi) nicht zu scharfe, trocknende Arzneimittel verwenden;

2. calor (,Hitze“, re) kühlende, Toxisches herauslösende Arzneimittel sollten nicht im Übermaß verwendet werden;

3. den richtigen Zeitpunkt zur Nutzung von suppletierenden Arzneimitteln finden und dabei nicht zu befeuchtende und klebrige Mitteln einsetzen;

4. dabei stets auf den Funktionszustand der oo. lienalis et stomachi (Fk „Milz“ und „Magen“, pi wei) achten.

An dieser Stelle möchte ich Ihnen Wu Youkes 吴又可 „Abhandlung über morbi temperati und epidemische Krankheiten“" (Wenyi lun 瘟疫论, 1642 verfasst) empfehlen, welches ein sorgfältiges Studium wert ist. Wir sollten nicht versuchen, die Rezepturen darin einfach zu kopieren, sondern die wahre Bedeutung der Rezeptur herauszulesen. Um diese Krankheit wirksam behandeln zu können, müssen wir die Theorien über algor laedens (schädigende „Kälte“, shanghan), morbi temperati („Wärme“-Erkrankungen, wenbing), innere Schädigungen der oo. lienalis et stomachi (Fk „Milz“ und „Magen“, pi wei) von Li Dongyuan, Erkrankungen des Yin von Wang Haogu 王好古 (ca. 1200 bis 1264) und die über eingestautes Xue gut verstehen. Durch die Integration dieser Theorien wird man in der Lage sein, mit dieser Krankheit besser umzugehen.

AC und SO: Vielen Dank für das Interview.

Zur interviewten Person Dr. Jin Zhao金钊, Ph.D., absolvierte sein Studium an der Chengdu University of TCM, sein Forschungsschwerpunkt sind die Klassiker der Chinesischen Medizin, insbesondere die sog. „Mitten-Denkschule“. Er ist (Co-)Autor zahlreicher Publikationen. Derzeit ist er Associate Professor der Chengdu University of Chinese Medicine und behandelt regelmäßig in verschiedenen Kliniken mit großem Zulauf und Erfolg. Dabei versucht er, die Erfahrungen der klassischen Chinesischen Medizin für eine Vielzahl von Erkrankungen, insbesondere aus den Fachgebieten Innere Medizin, Gynäkologie und Kinderheilkunde, nutzbar zu machen.

Zu den interviewenden Personen Andrea Crämer, Ärztin, Masterstudentin an der Chengdu University of Traditional Chinese Medicine (Lehre der Geschichte der Chinesischen Medizin und chinesischen Medizinalliteratur, Betreuer Prof. Liu Yuan 刘源) sowie Masterstudentin für TCM an der Technischen Universität München; Studentin von Prof. Jin Zhao.

Sebastian Oppitz, Dr. med. univ. M.Sc.; Facharzt für Allgemeinmedizin, Zusatzbezeichnung Akupunktur, Certified Physician of Chinese Medicine (SMS). Ausbildung in chinesischer Medizin bei der Internationalen Gesellschaft für Chinesische Medizin-SMS, sowie im Rahmen des Masterstudiengangs Traditionelle Chinesische Medizin an der Technischen Universität München.

2018 mehrmonatiger Studienaufenthalt an der Universität für Traditionelle Chinesische Medizin in Chengdu, China mit regelmäßiger Hospitation in der Praxis von Dr. Jin Zhao. Vorstandsmitglied der Internationalen Gesellschaft für Chinesische Medizin-SMS sowie des Berufsverbands Akupunktur. Privatärztliche Tätigkeit in der Praxis Prof. Hempen und Kollegen in München.

Hinweis des Verlags Der Verlag bleibt in Hinblick auf geografische Zuordnungen und Gebietsbezeichnungen in veröffentlichten Karten und Institutsadressen neutral. 